\title{
HARDY SPACES AND THE TWO-DIMENSIONAL EULER EQUATIONS WITH NONNEGATIVE VORTICITY
}

\author{
L. C. EVANS AND S. MÜLLER
}

\section{INTRODUCTION}

This paper is inspired by recent work of Delort [3] concerning solutions of the two-dimensional Euler equations with nonnegative vorticity. Delort's key observation, which we will explain in more detail in $\S 4$, is that whenever $\left\{\mathbf{v}_{\varepsilon}\right\}_{0<\varepsilon \leq 1}$ is an appropriate collection of velocity fields solving the two-dimensional incompressible Euler equations and

$$
\mathbf{v}_{\varepsilon} \rightarrow \mathbf{v} \text { weakly in } L_{\mathrm{loc}}^{2}, \quad \mathbf{v}_{\varepsilon} \rightarrow \mathbf{v} \text { a.e. as } \varepsilon \rightarrow 0,
$$

we can pass to limits in certain quadratic expressions involving $\mathbf{v}_{\varepsilon}$, provided the corresponding scalar vorticities are nonnegative. More precisely, we have

$$
\left\{\begin{array}{l}
v_{\varepsilon}^{1} v_{\varepsilon}^{2} \rightarrow v^{1} v^{2} \\
\left(v_{\varepsilon}^{1}\right)^{2}-\left(v_{\varepsilon}^{2}\right)^{2} \rightarrow\left(v^{1}\right)^{2}-\left(v^{2}\right)^{2}
\end{array}\right. \text { in the sense of distributions, }
$$

where $\mathbf{v}_{\varepsilon}=\left(v_{\varepsilon}^{1}, v_{\varepsilon}^{2}\right), \mathbf{v}=\left(v^{1}, v^{2}\right)$.

This assertion is remarkable, since it is not in general true that $\mathbf{v}_{\varepsilon} \rightarrow \mathbf{v}$ strongly in $L_{\text {loc }}^{2}$. The point is that the particular terms (1.2) (which arise naturally in Euler's equations) entail a kind of subtle cancellation to offset the possible strong concentrations of kinetic energy allowed by the weak convergence (1.1) .

Our intention here is to examine more closely this "concentration-cancellation" effect. The principal new result, Theorem 1, states that the expressions $\left\{v_{\varepsilon}^{1} v_{\varepsilon}^{2},\left(v_{\varepsilon}^{1}\right)^{2}-\left(v_{\varepsilon}^{2}\right)^{2}\right\}_{0<\varepsilon \leq 1}$ are "locally bounded" in the Hardy space $\mathscr{H}^{1}\left(\mathbb{R}^{2}\right)$; such terms are thus somewhat better than typical functions in $L_{\text {loc }}^{1}\left(\mathbb{R}^{2}\right)$. In addition, it turns out that this is enough information for us to provide a new proof of, and better insight into, the convergence (1.2); see Theorem 3.1 in $\S 3$.

To explain our assertions more precisely, we must introduce some terminology. Choose $\eta: \mathbb{R}^{n} \rightarrow \mathbb{R}$ to be any smooth function satisfying

$$
\operatorname{spt}(\eta) \subset B(0,1), \quad \int_{B(0,1)} \eta d x=1 .
$$

Received by the editors December 14, 1992 and, in revised form, April 5, 1993.

1991 Mathematics Subject Classification. Primary.

The first author was supported in part by NSF Grant DMS 92-03440. The second author was supported in part by NSF Grant DMS 90-02679 and SFB 256. 
If $f \in L^{1}\left(\mathbb{R}^{n}\right)$, we write

$$
f^{*}(x) \equiv \sup _{0<r<\infty}\left|\frac{1}{r^{n}} \int_{\mathbb{R}^{n}} f(y) \eta\left(\frac{x-y}{r}\right) d y\right|
$$

and define the Hardy, space

$$
\mathscr{H}^{1}\left(\mathbb{R}^{n}\right) \equiv\left\{f \in L^{1}\left(\mathbb{R}^{n}\right) \mid f^{*} \in L^{1}\left(\mathbb{R}^{n}\right)\right\} .
$$

According to Fefferman-Stein [8], this definition does not depend on the particular choice of $\eta$.

We will need a local version of (1.3). For $f \in L_{\text {loc }}^{1}\left(\mathbb{R}^{n}\right)$, we will hereafter set

$$
f^{* *}(x) \equiv \sup _{0<r \leq 1}\left|\frac{1}{r^{n}} \int_{\mathbb{R}^{n}} f(y) \eta\left(\frac{x-y}{r}\right) d y\right|
$$

and consider then the local Hardy space

$$
\mathscr{H}_{\mathrm{loc}}^{1}\left(\mathbb{R}^{n}\right) \equiv\left\{f \in L_{\mathrm{loc}}^{1}\left(\mathbb{R}^{n}\right) \mid f^{* *} \in L_{\mathrm{loc}}^{1}\left(\mathbb{R}^{n}\right)\right\}
$$

The point is that for $\mathscr{H}_{\text {loc }}^{1}$ we take the supremum only over radii $0<r \leq 1$, this in contrast to the supremum over all $r>0$ in the definition of $\mathscr{H}^{1}$. It will be useful to study also a related local Hardy space, introduced by Goldberg [12]:

$$
h^{1}\left(\mathbb{R}^{n}\right) \equiv\left\{f \in L^{1}\left(\mathbb{R}^{n}\right) \mid f^{* *} \in L^{1}\left(\mathbb{R}^{n}\right)\right\} .
$$

For a function $f \in h^{1}\left(\mathbb{R}^{n}\right)$, we set

$$
\|f\|_{h^{1}\left(\mathbb{R}^{n}\right)} \equiv\left\|f^{* *}\right\|_{L^{1}\left(\mathbb{R}^{n}\right)}
$$

Finally let $H_{\text {loc }}^{1}\left(\mathbb{R}^{n}\right)$ denote the usual Sobolev space of functions $f$ in $L_{\text {loc }}^{2}\left(\mathbb{R}^{n}\right)$, whose distributional first partial derivatives $f_{x_{1}}, \ldots, f_{x_{n}}$ belong as well to $L_{\text {loc }}^{2}\left(\mathbb{R}^{n}\right)$. We write $D f=D_{x} f=\left(f_{x_{1}}, \ldots, f_{x_{n}}\right)$.

Our main result concerns weakly superharmonic functions on $\mathbb{R}^{2}$.

Theorem 1.1. Let $u \in H_{\mathrm{loc}}^{1}\left(\mathbb{R}^{2}\right)$ be a weak solution of the PDE

$$
-\Delta u=\omega \text { in } \mathbb{R}^{2}
$$

where $\omega \in L_{\text {loc }}^{1}\left(\mathbb{R}^{2}\right)$ and

$$
\omega \geq 0
$$

Then

$$
u_{x_{1}} u_{x_{2}}, u_{x_{1}}^{2}-u_{x_{2}}^{2} \in \mathscr{H}_{\mathrm{loc}}^{1}\left(\mathbb{R}^{2}\right)
$$

In addition, for each $\phi \in C_{c}^{\infty}\left(\mathbb{R}^{2}\right)$ we have the estimate

$$
\left\|\phi u_{x_{1}} u_{x_{2}}\right\|_{h^{1}\left(\mathbb{R}^{2}\right)}+\left\|\phi\left(u_{x_{1}}^{2}-u_{x_{2}}^{2}\right)\right\|_{h^{1}\left(\mathbb{R}^{2}\right)} \leq C\|D u\|_{L^{2}(B(0, R))}^{2}
$$


for some constant $C$ and some radius $R$ depending only on $\phi$.

Our proof employs elementary singular integral-type computations to estimate directly the expression $\left(u_{x_{1}} u_{x_{2}}\right)^{* *}$ on compact sets. (Semmes [21] has recently discovered another proof, which employs more sophisticated Hardy space machinery.)

We will show by a counterexample in $\S 5$ that this assertion is in general false without the sign condition (1.8). (Interestingly, in the radial case the nonnegativity of $\omega$ is not required; see $\S 5$.)

The proof of Theorem 1.1 appears in $\S 2$. In $\S 3$ we present a local version of a theorem of Jones-Journé [13] asserting that a sequence which is bounded in $\mathscr{H}^{1}\left(\mathbb{R}^{n}\right)$ and converges a.e. in fact converges in the distribution sense. Section 4 rapidly recounts the fundamentals of two-dimensional incompressible flow and then explains an application of the theory in $\S \S 1-3$ to recover Delort's convergence theorem. Finally we collect some additional comments and examples in $\S 5$.

It is worth stressing that our full-blown program in $\S \S 2-3$ is not really required for the single application to Euler's equations-Delort employs much more direct and elementary arguments. Our real intention is rather to try to gain some further insight into the analytical behavior and physical significance of the nonlinear term $v^{1} v^{2}$ and its rotated counterpart $\left(v^{1}\right)^{2}-\left(v^{2}\right)^{2}$. A vague tenet of compensated compactness theory (cf. [7, Chapter 5]) holds that "physically natural" nonlinear terms in PDE should have better analytic properties than similar, but nonphysical, terms. We surely have here an instance of this phenomenon but are also currently lacking any really deep insight. A hope, as argued for other examples in the important paper Coifman, Lions, Meyer, and Semmes [2], is that Hardy space methods will continue to provide further clarification.

This work was initiated during the second author's visit to the Mathematical Sciences Research Institute at Berkeley, as part of the 1990-91 Special Year on PDE and Continuum Mechanics.

\section{Proof of Theorem 1.1}

Proof. (1) Fix $R>8$. Let $B(0, R)$ denote the closed ball with center 0 and radius $R$, and set

$$
v(x) \equiv \frac{-1}{2 \pi} \int_{B(0, R)} \omega(y) \log (|x-y|) d y .
$$

Then

$$
w \equiv u-v \text { is harmonic within } B(0, R)
$$

and

$$
v_{x_{i}}(x)=\frac{-1}{2 \pi} \int_{B(0, R)} \omega(y) \frac{x_{i}-y_{i}}{|x-y|^{2}} d y \quad(i=1,2) .
$$

(2) Choose $\eta \in C_{c}^{\infty}\left(\mathbb{R}^{n}\right)$ satisfying

$$
\operatorname{spt}(\eta) \subset B(0,1), \quad \int_{B(0,1)} \eta d x=1, \quad \eta \geq 0 .
$$


Let us also fix any point $x_{0} \in \mathbb{R}^{2}$. Consider then for $0<r \leq 1$ the expression

$$
\begin{aligned}
A \equiv \frac{1}{r^{2}} \int_{\mathbb{R}^{2}} v_{x_{1}}(x) v_{x_{2}}(x) \eta\left(\frac{x-x_{0}}{r}\right) d x & =\frac{1}{4 \pi^{2} r^{2}} \int_{B(0, R)} \int_{B(0, R)} \omega(y) \omega(z) \\
& \quad \times\left(\int_{\mathbb{R}^{2}} \frac{x_{1}-y_{1}}{|x-y|^{2}} \frac{x_{2}-z_{2}}{|x-z|^{2}} \eta\left(\frac{x-x_{0}}{r}\right) d x\right) d y d z,
\end{aligned}
$$

according to (2.3). (The interchange of integrations is valid, since the function $g(x) \equiv \int_{B(0, R)} \frac{\omega(y)}{|x-y|} d y$ belongs to $L_{\text {loc }}^{2}\left(\mathbb{R}^{2}\right)$; see (2.20).) We change variables by replacing $\left(x-x_{0}\right) / r$ with $x,\left(y-x_{0}\right) / r$ with $y$, and $\left(z-x_{0}\right) / r$ with $z$ to discover

$$
\begin{aligned}
A=\frac{r^{2}}{4 \pi^{2}} \int_{B\left(-x_{0} / r, R / r\right)} & \int_{B\left(-x_{0} / r, R / r\right)} \omega\left(x_{0}+r y\right) \omega\left(x_{0}+r z\right) \\
& \times\left(\int_{\mathbb{R}^{2}} \frac{x_{1}-y_{1}}{|x-y|^{2}} \frac{x_{2}-z_{2}}{|x-z|^{2}} \eta(x) d x\right) d y d z .
\end{aligned}
$$

Finally, define

$$
B \equiv \int_{\mathbb{R}^{2}} \frac{x_{1}-y_{1}}{|x-y|^{2}} \frac{x_{2}-z_{2}}{|x-z|^{2}} \eta(x) d x
$$

for fixed $y, z \in \mathbb{R}^{2}, y \neq z$.

(3) We assert

$$
|B| \leq C(1+|y|)^{-1}(1+|z|)^{-1} \quad\left(y, z \in \mathbb{R}^{2}, y \neq z\right)
$$

for some constant $C$, depending only on $\eta$. The proof is consequence of standard singular integral calculations (cf. Stein [22]) and so is only sketched below.

Case 1. $|y| \geq 2$ or $|z| \geq 2$. If $|y| \geq 2$, then

$$
|B| \leq \frac{C}{1+|y|} \int_{B(0,1)} \frac{1}{|x-z|} d x \leq C(1+|y|)^{-1}(1+|z|)^{-1} .
$$

If $|z| \geq 2$, a similar estimate obtains

Case 2. $|y| \leq 2$ and $|z| \leq 2$. In this situation, we write

$$
\begin{aligned}
B= & \eta(y) \int_{B(0,2)} \frac{x_{1}-y_{1}}{|x-y|^{2}} \frac{x_{2}-z_{2}}{|x-z|^{2}} d x \\
& +\int_{B(0,2)} \frac{x_{1}-y_{1}}{|x-y|^{2}} \frac{x_{2}-z_{2}}{|x-z|^{2}}(\eta(x)-\eta(y)) d x \\
& \equiv B_{1}+B_{2} .
\end{aligned}
$$

Clearly

$$
\left|B_{2}\right| \leq C(1+|y|)^{-1}(1+|z|)^{-1} .
$$


To estimate the term $B_{1}$, we examine two further possibilities:

Subcase 1. $|y|,|z| \leq 2,|y-z| \leq \frac{1}{2}(2-|y|)$. Then

$$
\begin{aligned}
B_{1}= & \eta(y) \int_{B(y, 2|y-z|)} \frac{x_{1}-y_{1}}{|x-y|^{2}} \frac{x_{2}-z_{2}}{|x-z|^{2}} d x \\
& +\eta(y) \int_{B(y, 2-|y|)-B(y, 2|y-z|)} \frac{x_{1}-y_{1}}{|x-y|^{2}} \frac{x_{2}-z_{2}}{|x-z|^{2}} d x \\
& +\eta(y) \int_{B(0,2)-B(y, 2-|y|)} \frac{x_{1}-y_{1}}{|x-y|^{2}} \frac{x_{2}-z_{2}}{|x-z|^{2}} d x \\
\equiv & C_{1}+C_{2}+C_{3} .
\end{aligned}
$$

We first estimate

$$
\begin{aligned}
\left|C_{1}\right| & \leq C \int_{B(y, 2|y-z|)} \frac{1}{|x-y|} \frac{1}{|x-z|} d x \\
& =C \int_{B(0,2)} \frac{1}{|x|} \frac{1}{|x-v|} d x \quad(v=z-y /|z-y|) \\
& \leq C .
\end{aligned}
$$

Now

$$
\begin{aligned}
C_{2} & =\eta(y) \int_{B(y, 2-|y|)-B(y, 2|y-z|)} \frac{x_{1}-y_{1}}{|x-y|^{2}} \frac{x_{2}-z_{2}}{|x-z|^{2}} d x \\
& =\eta(y) \int_{B(0,2-|y|)-B(0,2|w|)} \frac{x_{1}}{|x|^{2}}\left(\frac{x_{2}-w_{2}}{|x-w|^{2}}-\frac{x_{2}}{|x|^{2}}\right) d x \quad(w=z-y) .
\end{aligned}
$$

Since

if $|x| \geq 2|w|$

$$
\left|\frac{x-w}{|x-w|^{2}}-\frac{x}{|x|^{2}}\right| \leq C \frac{|w|}{|x|^{2}}
$$

$$
\left|C_{2}\right| \leq C|w| \int_{B(0,2-|y|)-B(0,2|w|)} \frac{1}{|x|^{3}} d x \leq C .
$$

Finally, note $\eta(y)=0$ if $|y| \geq 1$, and $2-|y| \geq 1$ if $|y| \leq 1$. Thus

$$
\left|C_{3}\right| \leq C \int_{B(0,2)-B(y, 1)} \frac{1}{|x-y|} \frac{1}{|x-z|} d x \leq C .
$$

Combining this calculation with $(2.11),(2.12)$, and (2.14) we deduce

$$
\left|B_{1}\right| \leq C \leq C(1+|y|)^{-1}(1+|z|)^{-1} .
$$

Subcase 2. $|y|,|z| \leq 2,|y-z| \geq \frac{1}{2}(2-|y|)$. In this situation, we can estimate

$$
\left|B_{1}\right| \leq C \eta(y)|\log | y-z||+C \text {. }
$$

But because $\frac{1}{2}(2-|y|) \leq|y-z| \leq 4$, we have

$$
\left|B_{1}\right| \leq C \eta(y)\left|\log \left(1-\frac{|y|}{2}\right)\right|+C \leq C .
$$


Consequently (2.15) holds in this subcase as well.

Combining at last $(2.8),(2.9),(2.10)$, and (2.15) we obtain estimate (2.7) .

(4) Return now to $(2.5),(2.6)$. In view of the bound (2.7) and the nonnegativity of $\omega$, we have

$$
\begin{aligned}
|A| & \leq C r^{2} \int_{B\left(-x_{0} / r, R / r\right)} \int_{B\left(-x_{0} / r, R / r\right)} \frac{\omega\left(x_{0}+r y\right)}{1+|y|} \frac{\omega\left(x_{0}+r z\right)}{1+|z|} d y d z \\
& =C\left(r \int_{B\left(-x_{0} / r, R / r\right)} \frac{\omega\left(x_{0}+r y\right)}{1+|y|} d y\right)^{2} .
\end{aligned}
$$

We restore the original variables by replacing $x_{0}+r y$ with $y$ :

$$
|A| \leq C\left(\int_{B(0, R)} \frac{\omega(y)}{r+\left|y-x_{0}\right|} d y\right)^{2}
$$

Since $\omega \geq 0$,

$$
\left(v_{x_{1}} v_{x_{2}}\right)^{* *}\left(x_{0}\right)=\sup _{0<r \leq 1}|A| \leq C\left(\int_{B(0, R)} \frac{\omega(y)}{\left|y-x_{0}\right|} d y\right)^{2} .
$$

(5) We next write

$$
g\left(x_{0}\right) \equiv \int_{B(0, R)} \frac{\omega(y)}{\left|y-x_{0}\right|} d y \quad\left(x_{0} \in \mathbb{R}^{2}\right)
$$

and claim

$$
g \in L_{\mathrm{loc}}^{2}\left(\mathbb{R}^{2}\right) .
$$

To establish this, first choose a cutoff function $\zeta \in C_{c}^{\infty}\left(\mathbb{R}^{n}\right)$, with $0 \leq \zeta \leq 1$, $\zeta=1$ on $B(0, R), \zeta \equiv 0$ on $\mathbb{R}^{2}-B(0,2 R),|D \zeta| \leq C / R$. Choose also $\lambda_{\varepsilon} \in$ $C^{\infty}\left(\mathbb{R}^{n}\right)$ satisfying $0 \leq \lambda_{\varepsilon} \leq 1, \lambda_{\varepsilon}=0$ on $B\left(x_{0}, \varepsilon\right), \lambda_{\varepsilon}=1$ on $\mathbb{R}^{2}-B\left(x_{0}, 2 \varepsilon\right)$, $\left|D \lambda_{\varepsilon}\right| \leq C / \varepsilon$. Since $-\Delta u=\omega$ in $\mathbb{R}^{2}$ in the weak sense,

$$
\begin{aligned}
\int_{\mathbb{R}^{2}} \zeta \lambda_{\varepsilon} \frac{\omega}{\left|y-x_{0}\right|} d y & =\int_{\mathbb{R}^{2}} D u \cdot D\left(\frac{\zeta \lambda_{\varepsilon}}{\left|y-x_{0}\right|}\right) d y \\
& =-\int_{\mathbb{R}^{2}} \zeta \lambda_{\varepsilon} D u \cdot \frac{\left(y-x_{0}\right)}{\left|y-x_{0}\right|^{3}} d y+\int_{\mathbb{R}^{2}} D u \cdot \frac{D\left(\zeta \lambda_{\varepsilon}\right)}{\left|y-x_{0}\right|} d y .
\end{aligned}
$$

Consequently

$$
\begin{aligned}
\int_{\mathbb{R}^{2}} \zeta \lambda_{\varepsilon} \frac{\omega}{\left|y-x_{0}\right|} d y \leq & -\int_{\mathbb{R}^{2}-B\left(x_{0}, 2 \varepsilon\right)} \zeta D u \cdot \frac{\left(y-x_{0}\right)}{\left|y-x_{0}\right|^{3}} d y \\
& +C M\left(|D u|_{B(0,2 R)}\right)\left(x_{0}\right)+C \int_{B(0,2 R)}|D u| \frac{1}{\left|y-x_{0}\right|} d y,
\end{aligned}
$$


where $M(\cdot)$ denotes the Hardy-Littlewood maximal function. Letting $\varepsilon \rightarrow 0$, we see

$$
\begin{aligned}
0 \leq g\left(x_{0}\right) \leq & \int_{\mathbb{R}^{2}} \zeta \frac{\omega}{\left|y-x_{0}\right|} d y \\
\leq & -\int_{\mathbb{R}^{2}} \zeta D u \cdot \frac{\left(y-x_{0}\right)}{\left|y-x_{0}\right|^{3}} d y \\
& +C M\left(|D u|_{B(0,2 R)}\right)\left(x_{0}\right)+C \int_{B(0,2 R)}|D u| \frac{1}{\left|y-x_{0}\right|} d y \\
& \equiv g_{1}\left(x_{0}\right)+g_{2}\left(x_{0}\right)+g_{3}\left(x_{0}\right) .
\end{aligned}
$$

The first integral in the last term is interpreted in the sense of principal value.

As $y /|y|^{3}$ is a Calderon-Zygmund kernel (cf. Stein [22]),

$$
\left\|g_{1}\right\|_{L^{2}\left(\mathbb{R}^{n}\right)} \leq C\|\zeta D u\|_{L^{2}\left(\mathbb{R}^{2}\right)} \leq C\|D u\|_{L^{2}(B(0,2 R))} .
$$

Furthermore, the maximal function preserves $L^{2}$; whence,

$$
\left\|g_{2}\right\|_{L^{2}\left(\mathbb{R}^{n}\right)} \leq C\|D u\|_{L^{2}(B(0,2 R))} .
$$

Finally $|y|^{-1} \in L_{\mathrm{loc}}^{1}\left(\mathbb{R}^{2}\right)$, so $g_{3} \in L_{\mathrm{loc}}^{2}\left(\mathbb{R}^{2}\right)$. In particular,

$$
\left\|g_{3}\right\|_{L^{2}\left(B\left(0, \frac{R}{2}\right)\right)} \leq C(R)\|D u\|_{L^{2}(B(0,2 R))} .
$$

In view of (2.21)-(2.24), assertion (2.20) is valid. Furthermore, utilizing (2.18), (2.19), and (2.21)-(2.24), we obtain the estimate

$$
\left\|\left(v_{x_{1}} v_{x_{2}}\right)^{* *}\right\|_{L^{1}\left(B\left(0, \frac{R}{2}\right)\right)} \leq C(R)\|D u\|_{L^{2}(0,2 R)}^{2} .
$$

(5) Now recall from (2.2) that $w=u-v$ is harmonic and thus smooth, within $B(0, R)$. Consequently if $x_{0} \in B(0, R / 2)$,

$$
\begin{aligned}
\left(w_{x_{1}} w_{x_{2}}\right)^{* *}\left(x_{0}\right) & =\sup _{0<r \leq 1}\left|\frac{1}{r^{2}} \int_{\mathbb{R}^{2}} w_{x_{1}} w_{x_{2}} \eta\left(\frac{x-x_{0}}{r}\right) d x\right| \\
& \leq C \sup _{0<r \leq 1} f_{B\left(x_{0}, r\right)}|D w|^{2} d x \\
& \leq C \sup _{B(0,3 R / 4)}|D w|^{2} .
\end{aligned}
$$

As $w$ is harmonic,

$$
\begin{aligned}
\sup _{B(0,3 R / 4)}|D w| & \leq C f_{B(0, R)}|w-\lambda| d x \\
& \leq C f_{B(0, R)}|u-\lambda|+|v| d x
\end{aligned}
$$

for each $\lambda \in \mathbb{R}$. Taking $\lambda=f_{B(0, R)} u d x$, we deduce, using Poincaré's inequality and $(2.1)$,

$$
\sup _{B(0,3 R / 4)}|D w| \leq C(R)\left(\|D u\|_{L^{2}(B(0, R))}+\|\omega\|_{L^{1}(B(0, R))}\right) .
$$


Utilizing then (2.26) we find

$$
\left\|\left(w_{x_{1}} w_{x_{2}}\right)^{* *}\right\|_{L^{1}(B(0, R / 2))} \leq C(R)\left(\|D u\|_{L^{2}(B(0, R))}^{2}+\|\omega\|_{L^{1}(B(0, R))}^{2}\right) .
$$

Similarly, if $x_{0} \in B(0, R / 2)$,

$$
\begin{aligned}
\left(w_{x_{1}} v_{x_{2}}\right)^{* *}\left(x_{0}\right)= & \sup _{0<r \leq 1}\left|\frac{1}{r^{2}} \int_{\mathbb{R}^{2}} w_{x_{1}} v_{x_{2}} \eta\left(\frac{x-x_{0}}{r}\right) d x\right| \\
\leq & C \sup _{0<r \leq 1} f_{B\left(x_{0}, r\right)}|D w|^{2}+|D w \| D u| d x \\
\leq & C(R)\left(\|D u\|_{L^{2}(B(0, R))}^{2}+\|\omega\|_{L^{1}(B(0, R))}^{2}\right) \\
& +C(R)\left(\|D u\|_{L^{2}(B(0, R))}+\|\omega\|_{L^{1}(B(0, R))}\right) \\
& +M\left(\mid D u \|_{B(0, R)}\right)\left(x_{0}\right),
\end{aligned}
$$

where we used (2.27). Hence

$$
\left\|\left(w_{x_{1}} v_{x_{2}}\right)^{* *}\right\|_{L^{1}(B(0, R / 2))} \leq C(R)\left(\|D u\|_{L^{2}(B(0, R))}^{2}+\|\omega\|_{L^{1}(B(0, R))}^{2}\right) .
$$

The same argument shows

$$
\left\|\left(v_{x_{1}} w_{x_{2}}\right)^{* *}\right\|_{L^{1}(B(0, R / 2))} \leq C(R)\left(\|D u\|_{L^{2}(B(0, R))}^{2}+\|\omega\|_{L^{1}(B(0, R))}^{2}\right) .
$$

Combining now (2.25), (2.27), (2.29), and (2.30) we obtain the bound

$$
\left\|\left(u_{x_{1}} u_{x_{2}}\right)^{* *}\right\|_{L^{1}(B(0, R / 2))} \leq C(R)\left(\|D u\|_{L^{2}(B(0,2 R))}^{2}+\|\omega\|_{L^{1}(B(0, R))}^{2}\right) .
$$

Finally choose a cutoff function $\psi \in C_{c}^{\infty}\left(\mathbb{R}^{n}\right)$ satisfying

$$
\left\{\begin{array}{l}
0 \leq \psi \leq 1, \quad|D \psi| \leq C / R, \\
\psi=1 \text { on } B(0, R), \quad \psi=0 \text { on } \mathbb{R}^{2}-B(0,2 R) .
\end{array}\right.
$$

Since $u$ is a weak solution of (1.7) and $\omega \geq 0$, we have

$$
\begin{aligned}
\int_{B(0, R)} \omega d x \leq \int_{B(0,2 R)} \omega \psi d x & =\int_{B(0,2 R)} D u \cdot D \psi d x \\
& \leq \frac{C}{R} \int_{B(0,2 R)}|D u| d x .
\end{aligned}
$$

Thus

$$
\|\omega\|_{L^{1}(B(0, R))}^{2} \leq C\|D u\|_{L^{2}(B(0,2 R))}^{2} .
$$

This estimate and (2.31) imply

$$
\left\|\left(u_{x_{1}} u_{x_{2}}\right)^{* *}\right\|_{L^{1}(B(0, R / 2))} \leq C(R)\|D u\|_{L^{2}(B(0,2 R))}^{2}
$$

for each $R \geq 8$. Hence $u_{x_{1}} u_{x_{2}} \in \mathscr{H}_{\text {loc }}^{1}\left(\mathbb{R}^{n}\right)$.

Rotating variables by replacing $x_{1}$ with $\left(x_{1}+x_{2}\right) / \sqrt{2}, x_{2}$ with $\left(x_{1}-x_{2}\right) / \sqrt{2}$, we deduce as well $u_{x_{1}}^{2}-u_{x_{2}}^{2} \in \mathscr{H}_{\text {loc }}^{1}\left(\mathbb{R}^{n}\right)$. Finally, estimate (1.10) is a consequence of Lemma 5.1 in $\S 5$. 


\section{HARDY SPACES AND CONVERGENCE A.E}

This section discusses some connections between convergence a.e. and convergence in the distribution sense, for a sequence bounded in the local Hardy space $h^{1}\left(\mathbb{R}^{n}\right)$. Jones and Journé [13] have recently shown that a sequence which is bounded in $\mathscr{H}^{1}\left(\mathbb{R}^{n}\right)$ and converges a.e., in fact converges in the sense of distributions. (See also Coifman, Lions, Meyer, and Semmes [2] for a generalization.) We require a local version of this result, and, for variety, set forth a somewhat different approach.

Following Goldberg [12], we begin by defining a local version of the space BMO. For this, take $g \in L_{\text {loc }}^{1}\left(\mathbb{R}^{n}\right)$ and write

$$
(g)_{I}=f_{I} g d x=\frac{1}{|I|} \int_{I} g d x
$$

to denote the average of $g$ over any cube $I$ with edges parallel to the coordinate axis. Then

$$
\|g\|_{B M O} \equiv \sup _{I} f_{I}\left|g-(g)_{I}\right| d x
$$

and

$$
\|g\|_{b m o} \equiv \sup _{|I| \leq 1} f_{I}\left|g-(g)_{I}\right| d x+\sup _{|I| \geq 1} f_{I}|g| d x .
$$

We say $g \in \operatorname{BMO}\left(\mathbb{R}^{n}\right)$ if $\|g\|_{\mathrm{BMO}}<\infty$, and $g \in \operatorname{bmo}\left(\mathbb{R}^{n}\right)$, the space of functions of locally bounded mean oscillation on $\mathbb{R}^{n}$, provided $\|g\|_{\text {bmo }}<\infty$.

According to [12], $\left(h^{1}\right)^{*}=$ bmo. More precisely, there exists a constant $C$, depending only on $n$, such that

$$
\left|\int_{\mathbb{R}^{n}} f g d x\right| \leq c\|h\|_{h^{1}\left(\mathbb{R}^{n}\right)}\|g\|_{\mathrm{bmo}},
$$

for all $f \in h^{1}\left(\mathbb{R}^{n}\right), g \in L^{\infty}\left(\mathbb{R}^{n}\right) \cap \operatorname{bmo}\left(\mathbb{R}^{n}\right)$.

We will require below the following assertion, due to Garnett-Jones [10, 11] and Uchiyama [24, Corollary 1]:

Lemma 3.1. Let $\lambda>0$, and let $A, B \subset \mathbb{R}^{n}$ be measurable sets such that

$$
\min \left\{\frac{|I \cap A|}{|I|}, \frac{|I \cap B|}{|I|}\right\} \leq 2^{-2 n \lambda}
$$

for each cube $I \subset \mathbb{R}^{n}$. Then there exists a measurable function $\eta: \mathbb{R}^{n} \rightarrow \mathbb{R}$ satisfying

$$
0 \leq \eta \leq 1, \eta=1 \quad \text { a.e. on } A, \quad \eta=0 \quad \text { a.e. on } B,
$$

and

$$
\|\eta\|_{\mathrm{BMO}} \leq C / \lambda
$$

The constant $C$ depends only on $n$.

A nice proof is available in Garnett-Jones [11].

We demonstrate next that a sequence which converges a.e. and which is bounded in the local Hardy space $h^{1}$, in fact converges in the distribution sense. 
Theorem 3.1. Assume $\left\{f_{h}\right\}_{k=1}^{\infty}$ is bounded in $h^{1}\left(\mathbb{R}^{n}\right)$ and

$$
f_{k} \rightarrow f \text { a.e. }
$$

for $f \in L_{\mathrm{loc}}^{1}\left(\mathbb{R}^{n}\right)$. Then

$$
f_{k} \rightarrow f \text { in the sense of distributions. }
$$

Proof. (1) Fix $\psi \in C_{c}^{\infty}\left(\mathbb{R}^{n}\right)$. We must show

$$
\int_{\mathbb{R}^{n}} f_{k} \psi d x \rightarrow \int_{\mathbb{R}^{n}} f \psi d x \quad \text { as } k \rightarrow \infty .
$$

(2) Choose any $\varepsilon>0$. Since $f_{k} \rightarrow f$ a.e., there exists an open set $G \subset \mathbb{R}^{n}$ such that

$$
|G|<\varepsilon
$$

and

$$
f_{k} \rightarrow f \text { uniformly on }\left(\mathbb{R}^{n}-G\right) \cap \operatorname{spt}(\psi) .
$$

(3) Let $Q$ denote the cube centered at the origin with side length $l(Q)=L$. Fix $L \geq 4$ so large

$$
\operatorname{spt}(\psi) \subset \frac{1}{2} Q .
$$

We want to apply Lemma 3.1 to the sets $A \equiv G \cap \frac{1}{2} Q, B \equiv \mathbb{R}^{n}-Q$. Now if $I$ is any cube for which either $|I \cap A|=0$ or $|I \cap B|=0$, then (3.3) holds for all $\lambda>0$. If on the other hand, $|I \cap A|>0$ and $|I \cap B|>0$, then necessarily $l(I)>L / 4$. Consequently

$$
\frac{|I \cap A|}{|I|} \leq \frac{4^{n}|A|}{L^{n}} \leq \frac{4^{n}|G|}{L^{n}} \leq \frac{4^{n} \varepsilon}{L^{n}} .
$$

Now

provided

$$
4^{n} \varepsilon / L^{n}=2^{-2 n \lambda}
$$

We deduce that there exists $\eta=\eta_{\varepsilon}$ satisfying (3.4) and

$$
\lambda=-1+\frac{\left|\log _{2} \varepsilon\right|}{2 n}+\frac{\log _{2} L}{2} \geq \frac{\left|\log _{2} \varepsilon\right|}{2 n} .
$$

(4) Let $I=\mu Q, \mu>1$. Since $0 \leq \eta \leq 1$ on $Q, \eta=0$ on $\mathbb{R}^{n}-Q$, we have

According to (3.13)

$$
\left|(\eta)_{I}\right| \leq \frac{|Q|}{|I|}=\frac{1}{\mu^{n}} .
$$

$$
\begin{aligned}
\int_{\mathbb{R}^{n}}|\eta| d x & \leq\left|(\eta)_{I}\right|+\int_{I}\left|\eta-(\eta)_{I}\right| d x \\
& \leq \frac{1}{\mu^{n}}+|I|\|\eta\|_{\mathrm{BMO}} \\
& \leq C\left(\frac{1}{\mu^{n}}+\frac{\mu^{n}}{\left|\log _{2} \varepsilon\right|}\right) .
\end{aligned}
$$


Selecting $\mu=\left|\log _{2} \varepsilon\right|^{1 / 2 n}$, we deduce

$$
\int_{\mathbb{R}^{n}}|\eta| d x \leq \frac{C}{\left|\log _{2} \varepsilon\right|^{1 / 2}} .
$$

(5) We turn now to the proof of (3.8). Let us compute

$$
\begin{aligned}
\left|\int_{\mathbb{R}^{n}}\left(f_{k}-f\right) \psi d x\right| \leq & \int_{\mathbb{R}^{n}}\left|f_{k}-f\right|(1-\eta)|\psi| d x \\
& +\left|\int_{\mathbb{R}^{n}} f_{k} \eta \psi d x\right|+\int_{\mathbb{R}^{n}}|f \eta \psi| d x \\
& \equiv A_{k}^{1}+A_{k}^{2}+A_{k}^{3} .
\end{aligned}
$$

Since $f_{k} \rightarrow f$ uniformly on $\left(\mathbb{R}^{n}-G\right) \cap \operatorname{spt}(\psi)$ and $\eta=1$ on $G \cap \operatorname{spt}(\psi)$,

$$
\lim _{k \rightarrow \infty} A_{k}^{1}=0 \text {. }
$$

Owing to (3.2)

$$
A_{k}^{2} \leq\left\|f_{k}\right\|_{h^{1}\left(\mathbb{R}^{n}\right)}\|\eta \psi\|_{\mathrm{bmo}} \leq C\|\eta \psi\|_{\mathrm{bmo}}
$$

Now if $|I| \geq 1$,

$$
f_{I}|\eta \psi| d x \leq\|\psi\|_{L^{\infty}} \int_{I}|\eta| d x \leq \frac{C}{\left|\log _{2} \varepsilon\right|^{1 / 2}} \quad \text { [by (3.15) ]. }
$$

If $|I| \leq 1$ and $x_{0}$ is the center of $I, l$ its side length, then

$$
\begin{aligned}
f_{I} \mid \eta \psi & -\psi\left(x_{0}\right)(\eta)_{I} \mid d x \\
& \leq f_{I}\left|\eta \psi-\eta \psi\left(x_{0}\right)\right| d x+f_{I}\left|\psi\left(x_{0}\right)\left(\eta-(\eta)_{I}\right)\right| d x \\
& \leq \frac{\operatorname{Lip}(\psi)}{l^{n-1}} \int_{I}|\eta| d x+\|\psi\|_{L^{\infty}} f_{I}\left|\eta-(\eta)_{I}\right| d x \\
& \leq C\left(\int_{I}|\eta|^{n} d x\right)^{1 / n}+C\|\eta\|_{\mathrm{BMO}} \\
& \leq C\left(\int_{\mathbb{R}^{n}}|\eta| d x\right)^{1 / n}+C\|\eta\|_{\mathrm{BMO}} \\
& \leq \frac{C}{\left|\log _{2} \varepsilon\right|^{1 / 2 n}}[\text { by (3.13), (3.15)]. }
\end{aligned}
$$

Combining the two cases above, we deduce

$$
\|\eta \psi\|_{\mathrm{bmo}} \leq \frac{C}{\left|\log _{2} \varepsilon\right|^{1 / 2 n}}
$$

whence (3.18) forces

$$
A_{k}^{2} \leq \frac{C}{\left|\log _{2} \varepsilon\right|^{1 / 2 n}}
$$


Recalling (3.16) and (3.17) we deduce

$$
\limsup _{k \rightarrow \infty}\left|\int_{\mathbb{R}^{n}}\left(f_{k}-f\right) \psi d x\right| \leq \frac{C}{\left|\log _{2} \varepsilon\right|^{1 / 2 n}}+C \int_{\mathbb{R}^{n}}|f \eta \psi| d x .
$$

In view of (3.15) there exists a subsequence $\varepsilon_{j} \rightarrow 0$ for which

$$
\eta=\eta_{\varepsilon_{j}} \rightarrow 0 \text { a.e. }
$$

Since $f \in L_{\text {loc }}^{1}\left(\mathbb{R}^{n}\right)$ and $0 \leq \eta_{\varepsilon} \leq 1$, we deduce upon sending $\varepsilon=\varepsilon_{j} \rightarrow 0$

$$
\lim _{k \rightarrow \infty}\left|\int_{\mathbb{R}^{n}}\left(f_{k}-f\right) \psi d x\right|=0
$$

Our proof uses the auxiliary function $\eta$ to "shield" the expressions on the left-hand side of (3.8) from the failure of strong convergence on the set $G$. The standard technique of isolating PDE from sets of small capacity is discussed for instance in Evans [7, pp. 38-47] and Frehse [9]; in these settings the appropriate auxiliary function $\eta$ is small in some Sobolev space. Lemma 3.1 allows us rather to shield an expression from a set of small Lebesgue measure, with a function $\eta$ small in BMO. (See also the discussion in $\S 4$ following, concerning the DiPerna-Majda [4] "concentration-cancellation" method.)

\section{AN APPLiCATION to THE 2-D EULER EQUATIONS WITH NONNEGATIVE VORTICITY}

This section presents an application of Theorems 1.1 and 1.3 to fluid mechanics.

Let us recall Euler's equations for a two-dimensional incompressible, inviscid fluid:

$$
\begin{cases}\mathbf{v}_{t}+\mathbf{v} \cdot D \mathbf{v}=-D p & \\ \operatorname{div} \mathbf{v}=0 & \text { in } \mathbb{R}^{2} \times(0, \infty) .\end{cases}
$$

Here $\mathbf{v}=\left(v^{1}, v^{2}\right)$ represents the fluid velocity and $p$ the pressure. The symbol $D$ denotes the gradient in the spacial variables $\left(x_{1}, x_{2}\right)$. The corresponding scalar vorticity is

$$
\omega \equiv v_{x_{2}}^{1}-v_{x_{1}}^{2}
$$

which satisfies the transport equation

$$
\omega_{t}+\mathbf{v} \cdot D \omega=0 \text { in } \mathbb{R}^{2} \times(0, \infty) .
$$

Uitilizing (4.1) we deduce conservation of kinetic energy:

$$
\frac{d}{d t}\left(\frac{1}{2} \int_{\mathbb{R}^{2}}|\mathbf{v}|^{2} d x\right)=0
$$

and invoking (4.3) we also compute

$$
\frac{d}{d t}\left(\int_{\mathbb{R}^{2}} \Phi(\omega) d x\right)=0
$$


for any smooth (and, by approximation, continuous) $\Phi: \mathbb{R} \rightarrow \mathbb{R}$. These calculations are valid for any smooth solution $(\mathbf{v}, p)$ of (4.1) decaying sufficiently rapidly at infinity. The case $\Phi$ is the absolute value function is particularly interesting, as this allows for an initial vorticity distribution $\omega_{0}$ which is only summable or even only a measure. The situation $\omega_{0}$ is a (signed) onedimensional measure restricted to a curve in $\mathbb{R}^{2}$ corresponds to the evolution of a vortex sheet under the flow (4.1). In view of detailed computer studies of Krasny [15] (cf. Majda [18]) we can expect in this case extraordinarily complicated vortex sheet stretching and roll-up phenomena.

As an initial attempt to understand rigorously the structure of such flows, DiPerna and Majda [4-6] proposed the mathematical problem of passing to limits in a sequence of approximating flows, under the physically natural assumption of uniform kinetic energy and total vorticity bounds. More precisely, assume for each parameter $0<\varepsilon \leq 1,\left(\mathbf{v}_{\varepsilon}, p_{\varepsilon}\right)$ is, say, a smooth solution of

$$
\begin{cases}\mathbf{v}_{\varepsilon, t}+\mathbf{v}_{\varepsilon} \cdot D \mathbf{v}_{\varepsilon}=-D p_{\varepsilon} & \\ \operatorname{div} \mathbf{v}_{\varepsilon}=0 & \text { in } \mathbb{R}^{2} \times(0, \infty),\end{cases}
$$

$\mathbf{v}_{\varepsilon}=\left(v_{\varepsilon}^{1}, v_{\varepsilon}^{2}\right)$. Suppose also we have the uniform bounds

$$
\sup _{0<\varepsilon \leq 1} \sup _{t \geq 0} \int_{K}\left|\mathbf{v}_{\varepsilon}\right|^{2}+\left|\omega_{\varepsilon}\right| d x \equiv C(K)<\infty
$$

for each compact set $K \subset \mathbb{R}^{2}$, where

$$
\omega_{\varepsilon} \equiv v_{\varepsilon, x_{2}}^{1}-v_{\varepsilon, x_{2}}^{1} \text {. }
$$

We assume as well for each time $T>0$ and some $s>0$ :

$$
\begin{aligned}
& \left\{\mathbf{v}_{\varepsilon}\right\}_{0<\varepsilon \leq 1} \text { is uniformly Lipschitz continuous from }[0, T] \text { into } \\
& \text { the local negative Sobolev space } H_{\text {loc }}^{-s}\left(\mathbb{R}^{2}\right) \text {. }
\end{aligned}
$$

See DiPerna-Majda [6, $\S \mathrm{C}]$ for the existence of $\left(\mathbf{v}_{\varepsilon}, p\right)$ verifying (4.6)-(4.9). (The existence of classical solutions for nice initial data is proved in Yudovitch [25], Kato [14], etc.) Passing to a subsequence $\left\{\varepsilon_{j}\right\}_{j=1}^{\infty}$ if necessary, we may then suppose

$$
\mathbf{v}_{\varepsilon_{j}} \rightarrow \mathbf{v} \text { weakly in } L_{\text {loc }}^{2}\left(\mathbb{R}^{2} \times(0, \infty)\right) .
$$

The mathematical question now is whether the limit velocity field $\mathbf{v}$ is in fact a weak solution of Euler's equations (4.1), for some appropriate pressure $p$. This problem is subtle since it is not at all clear that we can use the weak convergence to justify passing to limits in any sense in the nonlinear, quadratic terms $\mathbf{v}_{\varepsilon} \cdot D \mathbf{v}_{\varepsilon}=\operatorname{div}\left(\mathbf{v}_{\varepsilon} \otimes \mathbf{v}_{\varepsilon}\right)$.

DiPerna-Majda [4] propose a "concentration-cancellation" procedure to resolve this problem. Their program is to show that (a) the failure of strong convergence of $\mathbf{v}_{\varepsilon}$ to $\mathbf{v}$ in $L^{2}$ occurs only within the "small concentration set" and that (b) if this concentration set is sufficiently small, appropriate test functions can be employed to "shield" the PDE from the failure of strong convergence. 
This procedure turns out to work quite well for steady, time-independent flows; see DiPerna-Majda [4] for details. (Evans [7, pp. 42-47] provided a much simpler proof, and F. Bethuel has communicated to us an even easier method employing the Coarea Formula.)

For the full time-dependent problem, however, the concentration-cancellation technique has not as yet been successful, owing primarily to the relatively poor estimates available to control changes of $\mathbf{v}_{\varepsilon}$ in time. DiPerna-Majda [4] present some calculations on the concentration set with respect to a kind of "cylindrical" Hausdorff measure (their result is misstated in [4]), and H. Lopes [17] has recently derived bounds using true Hausdorff measure. These estimates are however too weak to allow for passage to limits from (4.6) to (4.1). In this context, see also Alinhac [1] and Zheng [26].

Recently, however, J.-M. Delort [3] has resolved this problem in the case that the scalar vorticities are all nonnegative:

$$
\omega_{\varepsilon} \geq 0 \text { in } \mathbb{R}^{2} \times(0, \infty) .
$$

In view of (4.5) (with $\omega_{\varepsilon}$ replacing $\omega$ ) we note (4.10) is valid if the initial vorticities are nonnegative. Delort proved that if $(4.6),(4.7),(4.9)$, and (4.10) hold, then the weak limit of the $\mathbf{v}_{\varepsilon}$ is indeed a weak solution of Euler's equations (4.1). His proof does not utilize the idea of estimating "the size of the concentration set" but instead relies on a close investigation of the quadratic terms $v_{\varepsilon}^{1} v_{\varepsilon}^{2},\left(v_{\varepsilon}^{1}\right)^{2}-\left(v_{\varepsilon}^{2}\right)^{2}$. As noted in $\S 1$, Delort in fact proves

$$
\left\{\begin{array}{l}
v_{\varepsilon_{j}}^{1} v_{\varepsilon_{j}}^{2} \rightarrow v^{1} v^{2} \\
\left(v_{\varepsilon_{j}}^{1}\right)^{2}-\left(v_{\varepsilon_{j}}^{2}\right)^{2} \rightarrow\left(v^{1}\right)^{2}-\left(v^{2}\right)^{2}
\end{array} \text { in the sense of distributions in } \mathbb{R}^{2} \times(0, \infty) .\right.
$$

This is enough, since, remarkably, we can rewrite (4.6) so that only these nonlinear quantities appear. To see this, note first (4.6) implies

$$
\int_{0}^{\infty} \int_{\mathbb{R}^{2}} \boldsymbol{\xi}_{t} \cdot \mathbf{v}_{\varepsilon}+D \boldsymbol{\xi}:\left(\mathbf{v}_{\varepsilon} \otimes \mathbf{v}_{\varepsilon}\right) d x d t=0
$$

for each test function $\boldsymbol{\xi} \in C_{c}^{\infty}\left(\mathbb{R}^{2} \times(0, \infty) ; \mathbb{R}^{2}\right)$ with $\operatorname{div} \boldsymbol{\xi}=0$. Following DiPerna-Majda [4] we take

$$
\boldsymbol{\xi}=D^{\perp} \eta=\left(-\eta_{x_{2}}, \eta_{x_{1}}\right),
$$

where $\eta \in C_{c}^{\infty}\left(\mathbb{R}^{2} \times(0, \infty)\right)$. Inserting (4.13) into (4.12) we deduce

$$
\begin{aligned}
\int_{0}^{\infty} \int_{\mathbb{R}^{2}}-\eta_{x_{2} t} v_{\varepsilon}^{1}+\eta_{x_{1} t} v_{\varepsilon}^{2} & +\eta_{x_{1} x_{2}}\left(\left(v_{\varepsilon}^{2}\right)^{2}-\left(v_{\varepsilon}^{1}\right)^{2}\right) \\
& +\left(\eta_{x_{2} x_{2}}-\eta_{x_{1} x_{1}}\right)\left(v_{\varepsilon}^{1} v_{\varepsilon}^{2}\right) d x d t=0
\end{aligned}
$$

We let $\varepsilon=\varepsilon_{j} \rightarrow 0$ and invoke (4.11) to conclude

$$
\begin{aligned}
\int_{0}^{\infty} \int_{\mathbb{R}^{2}}-\eta_{x_{2} t} v^{1}+\eta_{x_{1} t} v^{2} & +\eta_{x_{1} x_{2}}\left(\left(v^{2}\right)^{2}-\left(v^{1}\right)^{2}\right) \\
& +\left(\eta_{x_{2} x_{2}}-\eta_{x_{1} x_{1}}\right)\left(v^{1} v^{2}\right) d x d t=0
\end{aligned}
$$


for all $\eta$ as above. Thus

$$
\int_{0}^{\infty} \int_{\mathbb{R}^{2}} \boldsymbol{\xi}_{t} \cdot \mathbf{v}+D \boldsymbol{\xi}:(\mathbf{v} \otimes \mathbf{v}) d x d t=0
$$

for all test fields $\boldsymbol{\xi}$ having the form (4.13). As any divergence free field can be so written, we see $\mathbf{v}$ is a weak solution of Euler's equations (4.1).

As noted in $\S 1$, our motivation for the theory developed in $\S \S 1-3$ is to understand better Delort's work. To demonstrate the connections, let us introduce for each $\varepsilon>0$ and $t \geq 0$ the velocity potential $\psi_{\varepsilon}$ satisfying

$$
\mathbf{v}_{\varepsilon}=D^{\perp} \psi_{\varepsilon}=\left(-\psi_{\varepsilon, x_{2}}, \psi_{\varepsilon, x_{1}}\right) \text { in } \mathbb{R}^{2}
$$

The incompressibility condition $\operatorname{div} \mathbf{v}_{\varepsilon}=0$ ensures the existence of $\psi_{\varepsilon}$. Utilizing (4.8) we compute

$$
-\Delta \psi_{\varepsilon}=\omega_{\varepsilon} \text { in } \mathbb{R}^{2}
$$

Owing to (4.14) and (4.7) we see $\psi_{\varepsilon} \in H_{\mathrm{loc}}^{1}\left(\mathbb{R}^{2}\right)$ for each $t \geq 0$. In view of the vorticity sign assumption (4.10), our Theorem 1.1 is applicable: for each $\phi \in C_{c}^{\infty}\left(\mathbb{R}^{2}\right)$

$$
\left\|\phi v_{\varepsilon}^{1} v_{\varepsilon}^{2}\right\|_{h^{1}\left(\mathbb{R}^{2}\right)}+\left\|\phi\left(\left(v_{\varepsilon}^{1}\right)^{2}-\left(v_{\varepsilon}^{2}\right)^{2}\right)\right\|_{h^{1}\left(\mathbb{R}^{2}\right)} \leq C\left\|\mathbf{v}_{\varepsilon}\right\|_{L^{2}(B(0, R))}^{2}
$$

for some constant $C$ and some radius $R$, depending only on $\phi$. Owing to the uniform estimates (4.7), we conclude

$$
\begin{aligned}
& \left\{\phi v_{\varepsilon}^{1} v_{\varepsilon}^{2}, \phi\left(\left(v_{\varepsilon}^{1}\right)^{2}-\left(v_{\varepsilon}^{2}\right)^{2}\right)\right\}_{0<\varepsilon \leq 1} \text { are uniformly bounded in } h^{1}\left(\mathbb{R}^{2}\right), \\
& \text { for each time } t \geq 0
\end{aligned}
$$

Now in addition;

$$
\mathbf{v}_{\varepsilon_{j}} \rightarrow \mathbf{v} \text { strongly in } L_{\text {loc }}^{p}\left(\mathbb{R}^{2} \times(0, \infty)\right)
$$

for each $1 \leq p<2$. Indeed, for each time $t \geq 0$, we can deduce from (4.7), (4.15), and (4.16) that $\left\{\mathbf{v}_{\varepsilon_{j}}(\cdot, t)\right\}_{j=1}^{\infty}$ is precompact in $L_{\mathrm{loc}}^{p}\left(\mathbb{R}^{2}\right)$, if $1 \leq$ $p<2$; see, for instance, Evans [7, pp. 39-40]. On the other hand, according to DiPerna-Majda [4, Lemma A.1] (4.7) and (4.9) imply the mappings $t \mapsto$ $\mathbf{v}_{\varepsilon_{j}}(\cdot, t)$ are uniformly Hölder continuous from $[0, T]$ into $L_{\mathrm{loc}}^{p}\left(\mathbb{R}^{2}\right)$, for each $1 \leq p<2$. The sequence $\left\{v_{\varepsilon_{j}}\right\}_{j=1}^{\infty}$ is thus precompact in $L_{\mathrm{loc}}^{p}\left(\mathbb{R}^{2} \times(0, \infty)\right)$ for $1 \leq p<2$; whence (4.19) follows.

Now in view of (4.19) we may assume, upon passing to a further subsequence if needs be, that

$$
\mathbf{v}_{\varepsilon_{j}} \rightarrow \mathbf{v} \text { a.e. in } \mathbb{R}^{2} \times(0, \infty),
$$

and thus

$$
\mathbf{v}_{\varepsilon_{j}} \rightarrow \mathbf{v} \text { a.e. in } \mathbb{R}^{2}, \text { for a.e. } t \geq 0
$$


But then Theorem 3.1 implies

$$
\left\{\begin{array}{l}
\phi v_{\varepsilon_{j}}^{1} v_{\varepsilon_{j}}^{2} \rightarrow \phi v^{1} v^{2} \\
\phi\left(\left(v_{\varepsilon_{j}}^{1}\right)^{2}-\left(v_{\varepsilon_{j}}^{1}\right)^{2}\right) \rightarrow \phi\left(\left(v^{1}\right)^{2}-\left(v^{2}\right)^{2}\right)
\end{array}\right. \text { in the sense of distributions. }
$$

This assumption is valid for a.e. $t \geq 0$ and each $\phi \in C_{c}^{\infty}\left(\mathbb{R}^{2}\right)$; Delort's theorem (4.11) follows.

Remarks. (i) The condition (4.9) of Lipschitz continuity into some negative Sobolev space is essential to deduce the a.e. convergence (4.20). Indeed J. Serrin has noted that the function

$$
\mathbf{v}(x, t)=f(t) D w(x)
$$

is an exact solution of Euler's equations for some appropriate pressure $p$, whenever $w: \mathbb{R}^{2} \rightarrow \mathbb{R}$ is harmonic and $f:[0, \infty) \rightarrow \mathbb{R}$ is any smooth function. Choosing a bounded but highly oscillatory collection $\left\{f_{\varepsilon}\right\}_{0<\varepsilon \leq 1}$, we can arrange the corresponding solutions $\left\{\mathbf{v}_{\varepsilon}\right\}_{0<\varepsilon \leq 1}, \mathbf{v}_{\varepsilon}=f_{\varepsilon} D w$, to satisfy (4.7) but not possess an a.e. convergent subsequence.

(ii) Scheffer in [19] has recently constructed an example of a nontrivial weak solution of Euler's equations with compact support in $\mathbb{R}^{2} \times(0, \infty)$. Hence the identity (4.14) for all smooth, divergence free $\phi$ should presumably be augmented with some additional condition to select the "physically correct" velocity field $\mathbf{v}$.

\section{EXTENSIONS AND COMMENTS}

We gather together in this concluding section some additional comments and observations.

A. Localization. As the definitions of $\mathscr{H}_{\mathrm{loc}}^{1}$ and $h^{1}$ are a bit awkward, it is convenient to record:

Lemma 5.1. Let $f \in \mathscr{H}_{\text {loc }}^{1}\left(\mathbb{R}^{n}\right)$. Suppose also $\phi \in C_{c}^{\infty}\left(\mathbb{R}^{n}\right)$, with $\operatorname{spt}(\phi) \subset$ $B(0, R)$. Then

$$
\phi f \in h^{1}\left(\mathbb{R}^{n}\right)
$$

and we have the estimate

$$
\|\phi f\|_{h^{1}\left(\mathbb{R}^{n}\right)} \leq C\left\|f^{* *}\right\|_{L^{1}(B(0, R+2))},
$$

the constant $C$ depending only on $\phi$ and $R$.

Proof. Observe first

$$
(\phi f)^{* *}(x)=\sup _{0<r \leq 1}\left|\frac{1}{r^{n}} \int_{\mathbb{R}^{n}} \eta\left(\frac{x-y}{r}\right) \phi(y) f(y) d y\right|
$$


vanishes if $x \in \mathbb{R}^{n}-B(0, R+1)$. If instead $x \in B(0, R+1)$, we have

$$
\begin{aligned}
(\phi f)^{* *}(x) \leq & |\phi(x)| f^{* *}(x) \\
& +\sup _{0<r \leq 1}\left|\frac{1}{r^{n}} \int_{B(x, r)} \eta\left(\frac{x-y}{r}\right)(\phi(y)-\phi(x)) f(y) d y\right| \\
\leq & C\left(f^{* *}(x)+\sup _{0<r \leq 1} \frac{1}{r^{n-1}} \int_{B(x, r)}|f(y)| d y\right) .
\end{aligned}
$$

Now, as E. Stein has pointed out to us, if $x \in B(0, R+1)$,

$$
\sup _{0<r \leq 1} \frac{1}{r^{n-1}} \int_{B(x, r)}|f(y)| d y \leq \int_{B(0,1)}|\tilde{f}(x-y)||y|^{1-n} d y \equiv g(x),
$$

where $\tilde{f}=\left.f\right|_{B(0, R+2)}$. But

$$
\|g\|_{L^{1}\left(\mathbb{R}^{n}\right)} \leq C\|f\|_{L^{1}(B(0, R+2))} .
$$

Consequently

$$
\begin{aligned}
\|\phi f\|_{h^{1}\left(\mathbb{R}^{n}\right)} & =\left\|(\phi f)^{* *}\right\|_{L^{1}(B(0, R+1))} \\
& \leq C\left\|f^{* *}\right\|_{L^{1}(B(0, R+1))}+C\|f\|_{L^{1}(B(0, R+2))} \leq C\left\|f^{* *}\right\|_{L^{1}(B(0, R+2))},
\end{aligned}
$$

since $|f| \leq f^{* *}$ a.e.

B. Vorticity changing sign. Our goal next is to construct a counterexample to Theorem 1.1 if $\omega$ changes sign. We will need

Lemma 5.2. There exists a function $u \in C_{c}^{\infty}\left(\mathbb{R}^{n}\right)$ such that

$$
\operatorname{spt}(u) \subset B(0,1), \quad \int_{\mathbb{R}^{2}} u_{x_{1}} u_{x_{2}} d x=1 .
$$

Proof. Let $f, g, \tilde{f}, \tilde{g} \in C_{c}^{\infty}(-1 / 2,1 / 2)$, and define

$$
v\left(x_{1}, x_{2}\right)=f\left(x_{1}\right) g\left(x_{2}\right), \quad w\left(x_{1}, x_{2}\right)=\tilde{f}\left(x_{1}\right) \tilde{g}\left(x_{2}\right), \quad u=v+w .
$$

Since

$$
\int_{\mathbb{R}^{2}} v_{x_{1}} v_{x_{2}} d x=\int_{\mathbb{R}^{2}} w_{x_{1}} w_{x_{2}} d x=0
$$

an integration by parts yields

$$
\begin{aligned}
\int_{\mathbb{R}^{2}} u_{x_{1}} u_{x_{2}} d x & =\int_{\mathbb{R}^{2}}\left(v_{x_{1}} w_{x_{2}}+v_{x_{2}} w_{x_{1}}\right) d x \\
& =2 \int_{\mathbb{R}^{2}} v_{x_{1}} w_{x_{2}} d x=2\left(\int_{\mathbb{R}} f^{\prime} \tilde{f} d x_{1}\right)\left(\int_{\mathbb{R}} g \tilde{g}^{\prime} d x_{2}\right) .
\end{aligned}
$$

Choosing $\tilde{f}=f^{\prime} \not \equiv 0, g=c \tilde{g}^{\prime} \not \equiv 0$ the lemma follows for a suitable choice of the constant $c$.

Lemma 5.3. There exists a sequence of functions $\left\{u_{\varepsilon}\right\}_{0<\varepsilon \leq 1} \subset C_{c}^{\infty}\left(\mathbb{R}^{2}\right)$ satisfying

$$
\operatorname{spt}\left(u^{\varepsilon}\right) \subset B(0,1), \quad \sup _{0<\varepsilon \leq 1}\left(\left\|u^{\varepsilon}\right\|_{H^{1}\left(\mathbb{R}^{2}\right)}+\left\|\Delta u^{\varepsilon}\right\|_{L^{1}\left(\mathbb{R}^{2}\right)}\right)<\infty,
$$


but

$$
\left\|u_{x_{1}}^{\varepsilon} u_{x_{2}}^{\varepsilon}\right\|_{h^{1}\left(\mathbb{R}^{2}\right)} \rightarrow+\infty \text { as } \varepsilon \rightarrow 0
$$

Proof. Choose $u$ as in Lemma 5.2, and write

$$
u^{\varepsilon}(x) \equiv u\left(\frac{x}{\varepsilon}\right), \quad f(x) \equiv\left(u_{x_{1}} u_{x_{2}}\right)(x), \quad f^{\varepsilon}(x) \equiv\left(u_{x_{1}}^{\varepsilon} u_{x_{2}}^{\varepsilon}\right)(x)=\frac{1}{\varepsilon^{2}} f\left(\frac{x}{\varepsilon}\right) \text {. }
$$

Then estimate (5.4) follows easily by scaling. Set

$$
f^{\varepsilon, * *}(x) \equiv \sup _{0<r \leq 1}\left|\frac{1}{r^{2}} \int_{B(0,1)} \eta\left(\frac{x-y}{r}\right) f^{\varepsilon}(y) d y\right| \quad(0<\varepsilon \leq 1),
$$

where we assume in addition to the usual hypotheses

$$
\eta=1 \text { on } B(0,1 / 2) \text {. }
$$

We now claim

$$
f^{\varepsilon, * *}(x) \geq \frac{1}{16|x|^{2}} \quad \text { for } \varepsilon<|x| \leq \frac{1}{4} .
$$

Indeed, taking $\varepsilon<|x| \leq \frac{1}{4}$ and writing $y=\varepsilon z, r=4|x|$ we have

$$
\begin{aligned}
f^{\varepsilon, * *}(x) & \geq \frac{1}{16|x|^{2}} \int_{B(0,1)} \eta\left(\frac{x-\varepsilon z}{4|x|}\right) f(z) d z \\
& =\frac{1}{16|x|^{2}} \int_{B(0,1)} f(z) d z d y \quad[\text { by (5.6)] } \\
& =\frac{1}{16|x|^{2}} \quad[\text { by (5.3)]. }
\end{aligned}
$$

This proves (5.7), from which (5.5) follows.

C. Radial solutions. The example in Lemma 5.3 shows our Theorem 1.1 fails in general without the nonnegativity condition on $\omega$. In the radial case, however, no such restriction is necessary:

Theorem 5.4. Let $u \in H_{\mathrm{loc}}^{1}\left(\mathbb{R}^{2}\right)$ be a weak solution of the PDE

$$
-\Delta u=\omega \text { in } \mathbb{R}^{2}
$$

with $\omega \in L_{\mathrm{loc}}^{1}\left(\mathbb{R}^{2}\right)$ and

$$
u(x)=u(r), \quad \omega(x)=\omega(r)
$$

for $r=|x|$. Then

$$
u_{x_{1}} u_{x_{2}}, u_{x_{1}}^{2}-u_{x_{2}}^{2} \in \mathscr{H}_{\mathrm{loc}}^{1}\left(\mathbb{R}^{2}\right) \text {. }
$$

Furthermore, for each $\phi \in C_{c}^{\infty}\left(\mathbb{R}^{2}\right)$ we have the estimate

$$
\begin{aligned}
& \left\|\phi u_{x_{1}} u_{x_{2}}\right\|_{h^{1}\left(\mathbb{R}^{2}\right)}+\left\|\phi\left(u_{x_{1}}^{2}-u_{x_{2}}^{2}\right)\right\|_{h^{1}\left(\mathbb{R}^{2}\right)} \\
& \leq C\left(\|D u\|_{L^{2}(B(0, R))}^{2}+\|\omega\|_{L^{1}(B(0, R))}^{2}\right)
\end{aligned}
$$


for some constant $C$ and some radius $R$, depending only on $\phi$.

Proof. We have the ODE

$$
-\frac{1}{r}\left(r u^{\prime}\right)^{\prime}=\omega
$$

It suffices to prove the estimates for $u_{x_{1}} u_{x_{2}}$, as those for $u_{x_{1}}^{2}-u_{x_{2}}^{2}$ follow then by performing a rotation. Let $\eta \in C_{c}^{\infty}(B(0,1)), \int_{B(0,1)} \eta d x=1, \eta \geq 0$. Define

$$
\begin{aligned}
f(x) & \equiv\left(u_{x_{1}} u_{x_{2}}\right)(x)=\frac{x_{1} x_{2}}{r^{2}}\left(u^{\prime}\right)^{2}(r), \\
A_{i} & \equiv\left\{x: r_{i-1}<|x| \leq r_{i}\right\}, r_{i} \equiv 2^{i} \quad(i \in \mathbb{Z}), \\
f_{i} & \equiv f \chi_{A_{i}} .
\end{aligned}
$$

We also write

$$
\begin{aligned}
& f^{* *}(x) \equiv \sup _{0<r \leq 1}\left|\frac{1}{r^{2}} \int_{\mathbb{R}^{2}} \eta\left(\frac{x-y}{r}\right) f(y) d y\right|, \\
& f_{i}^{* *}(x) \equiv \sup _{0<r \leq 1}\left|\frac{1}{r^{2}} \int_{\mathbb{R}^{2}} \eta\left(\frac{x-y}{r}\right) f_{i}(y) d y\right| \quad(i \in \mathbb{Z}) .
\end{aligned}
$$

Since $\omega \in L_{\text {loc }}^{1}\left(\mathbb{R}^{2}\right)$, the ODE (5.9) implies $v(r)=r u^{\prime}(r)$ is absolutely continuous on each interval $[0, R]$. In particular, if $r_{i-1} \leq r \leq r_{i}$,

$$
\left|v(r)-\int_{r_{i-1}}^{r_{i}} v d r\right| \leq \int_{r_{i-1}}^{r_{i}}\left|v^{\prime}\right| d r \leq \int_{r_{i-1}}^{r_{i}} r|\omega| d r .
$$

We easily verify (see, e.g., Latter [16]) that if $h \in L^{\infty}\left(\mathbb{R}^{2}\right)$, spt $h \subset B(0, r)$, and $\int_{\mathbb{R}^{2}} h d x=0$, then

$$
\left\|h^{* *}\right\|_{L^{1}\left(\mathbb{R}^{2}\right)} \leq\left\|h^{*}\right\|_{L^{1}\left(\mathbb{R}^{2}\right)} \leq C r^{2}\|h\|_{L^{\infty}\left(\mathbb{R}^{2}\right)}
$$

Thus

$$
\left\|f_{i}^{* *}\right\|_{L^{1}\left(\mathbb{R}^{2}\right)} \leq C r_{i}^{2}\left\|f_{i}\right\|_{L^{\infty}}
$$

Moreover

$$
f_{i+2}^{* *}=0 \text { on } B\left(0, r_{i+1}-1\right) \supset B\left(0, r_{i}\right) \text { if } i \geq 1
$$

Now

$$
r_{i}^{2}\left\|f_{i}\right\|_{L^{\infty}} \leq 4 \sup _{r_{i-1}<r \leq r_{i}} r^{2}\left|u^{\prime}(r)\right|^{2}
$$


and by $(5.10)$

$$
\begin{aligned}
\sup _{r_{i-1} \leq r \leq r_{i}} r\left|u^{\prime}(r)\right| & \leq C\left\{\frac{1}{r_{i}} \int_{r_{i-1}}^{r_{i}}\left|r u^{\prime}\right| d r+\int_{r_{i-1}}^{r_{i}}\left|\left(r u^{\prime}\right)^{\prime}\right| d r\right\} \\
& \leq C\left\{\frac{1}{r_{i}^{1 / 2}} \int_{r_{i-1}}^{r_{i}}\left|r^{1 / 2} u^{\prime}\right| d r+\int_{r_{i-1}}^{r_{i}} r|\omega| d r\right\} \\
& \leq C\left\{\left(\int_{r_{i-1}}^{r_{i}} r\left|u^{\prime}\right|^{2} d r\right)^{1 / 2}+\int_{r_{i-1}}^{r_{i}} r|\omega| d r\right\} \\
& \leq C\left(\left\{\int_{A_{i}}|D u|^{2} d x\right\}^{1 / 2}+\int_{A_{i}}|\omega| d x\right) .
\end{aligned}
$$

Combining (5.11) through (5.14) it follows that

$$
\begin{aligned}
\left\|f^{* *}\right\|_{L^{1}\left(B\left(0, r_{i}\right)\right)} & \leq \sum_{j=-\infty}^{i+2}\left\|f_{j}^{* *}\right\|_{L^{1}\left(\mathbb{R}^{2}\right)} \\
& \leq C\left(\int_{B\left(0, r_{i+1}\right)}|D u|^{2} d x+\left(\int_{B\left(0, r_{i+1}\right)}|\omega| d x\right)^{2}\right) .
\end{aligned}
$$

Hence

$$
u_{x_{1}} u_{x_{2}}=f \in \mathscr{H}_{\mathrm{loc}}^{1}\left(\mathbb{R}^{2}\right) .
$$

Using Lemma 5.1 we deduce estimate (5.8).

\section{REFERENCES}

1. S. Alinhac, Un phénomène de concentration évanescente pour des flots non-stationnaires incompressibles en dimension deux, preprint, 1989.

2. R. Coifman, P.-L. Lions, Y. Meyer, and S. Semmes, Compacité par compensation et espaces de Hardy, C.R. Acad. Sci. Paris Ser. I Math. 309 (1991), 945-949.

3. J.-M. Delort, Existence de nappes de tourbillon en dimension deux, J. Amer. Math. Soc. 4 (1991), 553-586.

4. R. DiPerna and A. Majda, Reduced Hausdorff dimension and concentration cancellation for two-dimensional incompressible flow, J. Amer. Math. Soc. 1 (1988), 59-95.

5. Oscillations and concentrations in weak solutions of the incompressible flow equations, Comm. Math. Phys. 108 (1987), 667-689.

6. Concentrations in regularizations for 2-D compressible flow, Comm. Pure Appl. Math. 40 (1987), 301-345.

7. L. C. Evans, Weak convergence methods for nonlinear partial differential equations, CBMS Regional Conf. Ser. in Math., no. 74, Amer. Math. Soc., Providence, RI, 1990.

8. C. Fefferman and E. Stein, $\mathscr{H}^{p}$ spaces of several variables, Acta. Math. 129 (1972), 137-193.

9. J. Frehse, Capacity methods in the theory of partial differential equations, Jber. Deutsch. Math.-Verein 84 (1982), 1-44.

10. J. Garnett and P. Jones, The distance in BMO to $L^{\infty}$, Ann. of Math. (2) 108 (1978), 373-393.

11. _ BMO from dyadic BMO, Pacific J. Math. 99 (1982), 351-371.

12. D. Goldberg, A local version of real Hardy spaces, Duke Math. J. 46 (1979), 27-42. 
13. P. Jones and J.-L. Journé, On weak convergence in $\mathscr{H}^{1}\left(\mathbb{R}^{2}\right)$, Proc. Amer. Math. Soc. (to appear).

14. T. Kato, On classical solutions of the two-dimensional non-stationary Euler equation, Arch. Rational Mech. Anal. 25 (1967), 188-200.

15. R. Krasny, Computation of vortex sheet roll-up in the Treffitz plane, J. Fluid Mech. (1988).

16. R. H. Latter, A characterization of $\mathscr{H}^{p}\left(\mathbb{R}^{n}\right)$ in terms of atoms, Studia Math. 62 (1973), 93-101.

17. H. Lopes, An estimate on the Hausdorff dimension of a concentration set for the incompressible Euler equations, Thesis, UC Berkeley, 1991.

18. A. Majda, Vorticity and mathematical theory of incompressible fluid flow, Comm. Pure Appl. Math. 39 (1989), 187-220.

19. V. Scheffer, An inviscid flow with compact support in space-time, preprint, 1992.

20. F. Schulz, Regularity theory for quasilinear elliptic systems and Monge-Ampere equations in two dimensions, Lecture Notes in Math., vol. 1445, Springer, New York, 1990.

21. S. Semmes, A primer on Hardy spaces, and some remarks on a theorem of Evans and Müller, preprint, 1993.

22. E. Stein, Singular integrals and differentiability properties of functions, Princeton Univ. Press, Princeton, NJ, 1970.

23. A. Torchinsky, Real variable methods in harmonic analysis, Academic Press, New York, 1986.

24. A. Uchiyama, The construction of certain BMO functions and the corona problem, Pacific J. Math. 99 (1982), 183-204.

25. V. I. Yudovich, Non-Stationary flow of an ideal incompressible liquid, Zh. Vych. Math. 3 (1963), 1032-1066. (Russian)

26. Y. Zheng, Concentration-cancellation for the velocity fields in two dimensional incompressible fluid flows, Comm. Math. Phys. 135 (1991), 581-594.

27. __ Regularity of weak solutions to a two dimensional modified Dirac-Klein-Gordon system of equations, Comm. Math. Phys. 151 (1993), 67-87.

ABSTRACT. We prove that certain quadratic expressions involving the gradient of a weakly superharmonic function in $\mathbb{R}^{2}$ belong to a local Hardy space. As an application we provide a new proof of J.-M. Delort's convergence theorem for solutions of the two-dimensional Euler equations with vorticities of one sign.

Department of Mathematics, University of California, Berkeley, California 94720 MANY

SFB 256, Institut für Angewandte Mathematik, Beringstrasse 4, D5300 Bonn 1, Ger- 